Research Article

Cellular, Molecular and Developmental Genetics

\title{
Human DDX56 protein interacts with influenza A virus NS1 protein and stimulates the virus replication
}

\author{
Ayşegül Pirinçal $^{1}$ (D) and Kadir Turan² (D) \\ ${ }^{I}$ Marmara University, Institute of Health Sciences, Istanbul, Turkey. \\ ${ }^{2}$ Marmara University, Faculty of Pharmacy, Department of Basic Pharmaceutical Sciences, Istanbul, Turkey.
}

\begin{abstract}
Influenza A viruses (IAV) are enveloped viruses carrying a single-stranded negative-sense RNA genome. Detection of host proteins having a relationship with IAV and revealing of the role of these proteins in the viral replication are of great importance in keeping IAV infections under control. Consequently, the importance of human DDX56, which is determined to be associated with a viral NS1 with a yeast two-hybrid assay, was investigated for IAV replication. The viral replication in knocked down cells for the DDX56 gene was evaluated. The NS1 was co-precipitated with the DDX56 protein in lysates of cells transiently expressing DDX56 and NS1 or infected with the viruses, showing that NS1 and DDX56 interact in mammalian cells. Viral NS1 showed a tendency to co-localize with DDX56 in the cells, transiently expressing both of these proteins, which supports the IP and two-hybrid assays results. The data obtained with in silico predictions supported the in vitro protein interaction results. The viral replication was significantly reduced in the DDX56-knockdown cells comparing with that in the control cells. In conclusion, human DDX56 protein interacts with the IAV NS1 protein in both yeast and mammalian cells and has a positive regulatory effect on IAV replication. However, the mechanism of DDX56 on IAV replication requires further elucidation.
\end{abstract}

Keywords: Influenza A viruses, NS1 protein, RNA helicase DDX56, virus-host interaction.

Received: May 22, 2020; Accepted: February 08, 2021.

\section{Introduction}

Influenza A viruses are enveloped viruses with a singlestranded RNA genome having a negative-polarity. The viral genome consisting of eight RNA segments encodes 11 different proteins (Nakajima, 1997). Non-structural proteins (NS1 and NS2) are synthesized through segment 8, the smallest segment of the viral genome (Lamb and Choppin, 1979). The viral NS1 protein is abundantly synthesized in infected cells, but it is not detected in the virions (Hale et al., 2008; Basu et al., 2009). The NS2 is synthesized via alternative splicing mechanisms through viral segment 8 . The NS1 and NS2 proteins share 10 amino acids in the amino-terminal region. The NS2 protein, which is found in the lowest amount in the virion, has a role in the transport of viral ribonucleoprotein particles (RNPs) with M1 protein and several cellular proteins (Senbas Akyazi et al., 2020). Therefore, it is also called a nuclear export protein (NEP) (Neumann et al., 2000; Kawaoka and Neumann, 2012). Unlike the NS2 protein, the most important function of NS1 is to cope with the antiviral effects of interferon response mechanisms, which are the intracellular defense system. Therefore, the NS1 protein plays a key role on efficient viral replication in infected cells (Kochs et al., 2007). The influenza A virus NS1 protein has an average of $26 \mathrm{kDa}$ molecular weight and consists of 230 or 237 amino acid residues depending on the virus type (Lin et al., 2007). This protein has the functional domains for both protein-protein and protein-RNA interactions.

Send correspondence to Kadir Turan. Marmara University, Faculty of Pharmacy, Department of Basic Pharmaceutical Sciences, Recep Tayyip Erdoğan Külliyesi, 34854, 4/A, Başıbüyük, Maltepe, Istanbul, Turkey. E-mail: kadirturan@ marmara.edu.tr.
The amino-terminal region of the NS1 protein, which contains 1-73 amino acid residues, is the RNA-binding domain and binds to various RNA molecules with low affinity. It has been reported that NS1 protein has a binding ability to the double stranded RNA (dsRNA) molecules formed during viral infection, U6 snRNA, which is a member of the protein/RNA (spliceosome) complex involved in the processing of RNA molecules and the poly-A structure consisting of adenine nucleotides (Hatada and Fukuda, 1992; Qiu et al., 1995). The binding of the NS1 protein via the RNA-binding domain to the dsRNA molecules is of great importance in terms of coping with host interferon defense mechanisms (Newby et al., 2007). The NS1 protein binds to the dsRNA molecules as a dimeric form (Wang W et al., 1999). The amino-terminal region of the NS1 protein is also important for binding to the several cellular proteins. This protein prevents the maturation and transport of cellular mRNAs to the cytoplasm by binding to the cleavage and polyadenylation specificity factor (CPSF) and polyA-binding (PABII) proteins (Nemeroff et al., 1998). In contrast, it shows a positive regulatory effect on viral mRNA translation by binding the 5'UTR region of viral mRNAs and PABPI (de la Luna et al., 1995; Burgui et al., 2003). The region covering the 73-230 amino acid residues of the NS1 protein is the carboxyl-terminal region in the homodimer structure, also known as the effector domain (Xia et al., 2009). It has been suggested that the C-terminal part of NS1 has a function in the regulation of IFN $\alpha / \beta$, tumor necrosis factor- $\alpha$ $(\mathrm{TNF}-\alpha)$ in macrophages infected with influenza A virus, IL-6, and the synthesis of chemokine ligand-3 (CCL3) while the N-terminal domain of the protein inhibits interleukin- $1 \beta$ (IL-1 $\beta$ ) and interleukin-18 (IL-18) synthesis (Stasakova et al., 2005; Lam et al., 2011). 
Like other RNA viruses, the influenza viruses use gene expression mechanisms and metabolites of the host cell to replicate by interacting with several cellular proteins. Therefore, the detection of the host cell proteins associated with viral proteins has great importance for the understanding of the viral pathogenesis. In this study, human proteins that may be related to influenza A virus NS1 protein were screened with the yeast two-hybrid method, an effective method for detecting protein interactions, and several candidate proteins previously not reported were identified. The human DDX56 protein, one of the candidate proteins interacting with viral NS1 protein in the yeast cells, was investigated in terms of interaction with NS1 and the effect on the replication of influenza A virus in the mammalian cells. The DDX56 protein, classified into the DEAD-box (Asp-Glu-Ala-Asp) protein family, is an ATP dependent RNA helicase enzyme that plays a role in the RNA metabolism (Zirwes et al., 2000). However, it was reported that the DDX56 protein affects the replication of some viruses such as the West Nile virus (WNV) (Xu et al., 2011; Xu and Hobman, 2012), FMDV (foot and mouth disease virus) (Fu et al., 2019a) and the Sendai virus (SeV) (Fu et al., 2019b) by directly interacting with the viral proteins or indirectly with host proteins. In this respect, it may be one of the cellular protein factors that positively regulate infections of influenza A viruses that undergo replication and transcription in the cell nucleus.

\section{Material and Methods}

\section{Cells, viruses, and antibodies}

HEK293 (Human embryonic kidney 293), HeLa (human epithelial carcinoma), and MDCK (Madin-Darby canine kidney) cells were used in transient transfection experiments and/or viral infections. The cells were cultured in Dulbecco's modified Eagle's medium (DMEM) supplemented with 10\% $(\mathrm{v} / \mathrm{v})$ heat-inactivated fetal calf serum (Gibco, USA), $2 \mathrm{mM}$ L-glutamine, $100 \mu \mathrm{g} / \mathrm{mL}$ streptomycin, $100 \mathrm{IU} / \mathrm{mL}$ penicillin, and $1.5 \mathrm{mg} / \mathrm{mL}$ sodium bicarbonate at $37^{\circ} \mathrm{C}$ in a humidified incubator with $5 \% \mathrm{CO}_{2}$.

Human influenza virus A/WSN/1933/H1N1 (WSN) and avian influenza virus A/duck/Pennsylvania/10.218/1984/H5N2 (DkPen) were propagated in MDCK cells and/or specific pathogen-free chicken embryos. The viral titer was measured using a standard plaque assay or hemagglutinin (HA) assay (Turan et al., 1996).

Monoclonal mouse anti-HA (Santa Cruz, \# sc-7392), polyclonal rabbit anti-NS1 (Invitrogen, \# PA5-322439), monoclonal mouse anti-actin (MyBioSource, \# MBS9400413), rabbit anti-M1 serum, horseradish peroxidase-conjugated second antibody against species-specific immunoglobulins [anti-mouse IgG-HRP (Invitrogen, \# 31420) and/or antirabbit IgG-HRP (Invitrogen, \# 31423)], Alexa-488conjugated goat anti-mouse IgG (Abcam, \# ab150117) and/ or Alexa-568-conjugated goat anti-rabbit IgG (Abcam, \# ab175471) antibodies were used in immunoblotting and/or immunofluorescence experiments.

\section{RNA extraction and first-strand cDNA synthesis}

In order for the PCR amplification of human influenza A virus $\mathrm{NS}_{\mathrm{W}}$ (WSN) cDNA, the avian influenza A virus $\mathrm{NS}_{\mathrm{D}}$ (Dk/Pen) cDNA, DDX56 cDNA and/or quantitation of the related transcripts with revers transcription-quantitative $\mathrm{PCR}$ (RT-qPCR), the total RNA was prepared from the cells with the RNeasy plus mini kit (Qiagen, Germany). cDNAs were prepared from $500 \mathrm{ng}$ total RNA derived from HeLa and/or HEK293 cells by using the Moloney murine leukemia virus reverse transcriptase (ReverTraAce, Toyobo Co., Ltd., Japan) and oligo(dT) as a primer for $60 \mathrm{~min}$ at $45^{\circ} \mathrm{C}$. The influenza A virus $\mathrm{NS}_{\mathrm{W}}$ cDNA and NS1 $1_{\mathrm{D}} \mathrm{cDNA}$ were obtained from virus-infected HeLa cells.

\section{Constructon of plasmid vectors}

All plasmids were constructed using PCR and standard subcloning techniques. The primers used for the PCRs are listed in Table 1.

\section{Mammalian cell expression plasmids}

For the construction of DDX56 expression vectors, the full length of the DDX56 gene consisting of 1644 bp was amplified from HEK293 cDNA with the phosphorylated primer pair DDX56-For and DDX56-Rev. PCR amplification was carried out with a thermostable DNA polymerase (KOD plus, Toyobo Co., Ltd., Japan). The PCR product was purified with an agarose gel extraction kit (QiaexII, Qiagen, Germany). For the construction of the human influenza A virus NS1 and avian influenza $A$ virus $\mathrm{NS}_{\mathrm{D}}$ expression vectors, NS1

Table 1 - Oligonucleotide primers used in this study.

\begin{tabular}{lr}
\hline Primers name & Sequence $\left(5^{\prime}-3\right.$ ') \\
\hline DDX56-For & ATCATGGAGGACTCTGAAGCACTG \\
DDX56-Rev & ATTCAGGAGGGCTTGGCTGTG \\
NS1/W-For & TTGAATTCGGAGGATCTGGAATGGATCCAAACACTGTGTC \\
NS1/W-Rev & TTGAATTCTCAAACTTCTGACCTAATTG \\
NS1/D-For & TTGAATTCGGAGGATCTGGAATGGACTCCAACACGATAAC \\
NS1/D-Rev & TTGAATTCTCAAACTTCTGACTCAACTC \\
DDX56/831-For & CTTGGAACAGTTCAGCATCC \\
DDX56/959-Rev & AGGACTTCAGCATCAGTTGC \\
ACTB-For & ATGGAGTCCTGTGGCATCC \\
ACTB-Rev & CCAGCACAATGAAGATCAAG \\
AD-Seq-For & AATACCACTACAATGGATGATGT \\
\hline
\end{tabular}


genes consisting of $693 \mathrm{bp}$ were amplified from virus-infected HeLa cDNA with primer pairs NS1/W-For and NS1/W-Rev or NS1/D-For and NS1/D-Rev. The PCR product was digested with EcoRI and purified with an agarose gel extraction kit.

In order to construct the pCHA-DDX56 plasmid encoding the HA-tagged DDX56 protein (H-DDX56), the DDX56 cDNA was cloned into pCHA (Nagata et al., 1998) plasmid digested with EcoRV (New England Biolabs, UK) and dephosphorylated with shrimp alkaline phosphatase (Thermo Fisher Scientific, USA). For the pCAGGS-NS $1_{w}$ plasmid vector construction, $\mathrm{NS}_{\mathrm{w}}$ cDNA was ligated with pCAGGS (Niwa et al., 1991) plasmid digested with EcoRI (New England Biolabs, UK) and dephosphorylated with SAP.

\section{Yeast cell expression plasmids}

In order to construct the $\mathrm{pGBD}-\mathrm{NS} 1_{\mathrm{w}}$ and $\mathrm{pGBD}$ NS1 $1_{\mathrm{D}}$ plasmid vectors, the pGBD-C1 (James et al., 1996) plasmid was digested with EcoRI, dephosphorylated with $\mathrm{SAP}$, and ligated with PCR amplified $\mathrm{NS} 1_{\mathrm{W}} \mathrm{cDNA}$ and $\mathrm{NS} 1_{\mathrm{D}}$ cDNA, respectively. For the pACT2-DDX56 plasmid vector construction, the PCR amplified DDX56 cDNA was ligated with pACT2 (Clontech, \# 638822) plasmid digested with BglII, blunted with a Klenow fragment (New England Biolabs, UK) and dephosphorylated with SAP. The nucleotide sequences of all plasmids were confirmed by DNA sequencing.

\section{Immunoblotting}

Immunoblotting analyses were used to detect the expression levels of DDX56 and viral proteins in transiently transfected and/or virus infected cells. Cell lysates were prepared using a RIPA buffer or SDS-PAGE sample buffer. The proteins in lysates were separated using $10 \%$ SDSPAGE and transferred to a polyvinylidene difluoride (PVDF) membrane. After blocking with 5\% skim milk, the membrane was incubated with the specific primary antibodies (mouse anti-HA, rabbit anti-NS1, mouse anti-actin and/or rabbit anti-M1 serum) overnight at $4{ }^{\circ} \mathrm{C}$ and followed by incubation with a horseradish peroxidase-conjugated second antibody [anti-mouse IgG-HRP and/or anti-rabbit IgG-HRP] for 45 minutes at room temperature. The proteins were visualized with an enhanced chemiluminescence detection kit (GE Healthcare, Italy).

\section{Yeast two-hybrid screening}

The yeast two-hybrid system based on the GAL4 transcription factor was used to identify host proteins that interact with influenza A virus NS1 protein. In brief, the Saccharomyces cerevisiae strain PJ69-4A(James et al., 1996) [MATa trp1-901 leu2-3,112 ura3-52 his3-200 gal4(delesyon) gal80(delesyon) LYS2::GAL1-HIS3 GAL2-ADE2 met2::GAL7lacZ] was grown in a YPAD medium. The pGBD-NS1 ${ }_{\mathrm{w}}$ plasmid coding GAL4-BD-NS $1_{\mathrm{w}}$ bait fusion protein was transformed into the yeast PJ69-4A strain by using the lithium acetate/polyethylene glycol (LiAc/PEG) and spread on synthetic dropout (SD) agar plates (without Trp) at $30{ }^{\circ} \mathrm{C}$. Then the transformants were selected on a SD(-Trp) plate and checked for the $\mathrm{NS1}_{\mathrm{w}}$ gene with PCR. The PJ69-4A yeast strain expressing the bait protein was transformed with the largescale cDNA library derived from HEK293 cells (Clontech,
\# 638826) and screened following the matchmaker twohybrid system protocol. Candidates for two hybrid interaction were selected on SD agar plates (without Ade, His, Leu, and Trp) for the primary screening and further tested by the $\beta$-galactosidase assay for the second screening. After rescue, the potential positive plasmids carrying cDNAs were isolated using a yeast plasmid DNA miniprep kit (Bio Basic, Canada) according to the manufacturer's instructions. Competent $E$. coli $\mathrm{DH} 5 \alpha$ were transformed with the plasmids isolated from yeast. Transformants were grown on Luria-Bertani agar plates containing $100 \mathrm{mg} / \mathrm{mL}$ ampicillin. Only the clones that were positive for all the reporters and confirmed to be positive by at least two independent tests were selected for specific interactions, sequenced, and identified with BLAST (Basic Local Alignment Search Tool) analysis.

\section{Re-transforming pACT2-DDX56 into the yeast cells and checking NS1 and DDX56 interaction}

To eliminate false-positive interactions and check the interaction NS1 between DDX56, pACT2-DDX56 plasmid coding a fusion of GAL4-AD-DDX56 or pACT2 plasmid (as a control) were transformed into the $S$. cerevisiae strain PJ69-4A containing the NS1 bait plasmid (pGBD-NS1 $1_{\mathrm{w}}$ or pGBD-NS1 $1_{\mathrm{D}}$ ) or pGBD-C1 (as a control) with the LiAc/ PEG. Double transformants were selected on SD agar plates (without Leu and Trp). To define the growth profiles, four colonies were cultured on SD agar plates (without Ade, His, Leu, and Trp) and tested for $\beta$-galactosidase enzyme activities.

\section{$\beta$-galactosidase assay}

Colonies defined the growth profiles were grown in a $5 \mathrm{~mL}$ SD medium (without Trp/Leu or Trp) at $30{ }^{\circ} \mathrm{C}$. The cells in $500 \mu \mathrm{L}$ of saturated culture were recovered with centrifugation and re-suspended in $300 \mu \mathrm{L}$ Z- buffer $(0.1$ $\mathrm{M}$ sodium phosphate $\mathrm{pH} 7.0,10 \mathrm{mM} \mathrm{KCl}, 1 \mathrm{mM} \mathrm{MgSO}_{4}$, and $0.35 \% \beta$-mercaptoethanol). The cells in suspensions were disintegrated in a freeze-thaw procedure repeated five times in liquid nitrogen. Then, $60 \mu \mathrm{L}$ o-nitrophenyl- $\beta$-dgalactopyranoside (ONPG) $(4 \mathrm{mg} / \mathrm{mL})$ substrate was added and incubated for 60 minutes at $37^{\circ} \mathrm{C}$. To stop the reactions $300 \mu \mathrm{L} \mathrm{Na}_{2} \mathrm{CO}_{3}(0.5 \mathrm{M})$ was added. The supernatants were recovered by centrifugation at $15000 \mathrm{rpm}$ for $5 \mathrm{~min}$, and then the absorbance of samples at $420 \mathrm{~nm}\left(\mathrm{OD}_{420}\right)$ was measured. The $\beta$-galactosidase activities were calculated from a standard curve plotted with a recombinant $\beta$-galactosidase enzyme (Roche).

\section{Co-immunoprecipitation assay}

Co-immunoprecipitation assays were used to confirm the interaction between NS1 and DDX56 in mammalian cells. The HEK293 cells $\left(5 \times 10^{5}\right)$ were seeded in a 6-well plate and incubated under standard culture conditions $\left(37^{\circ} \mathrm{C}\right.$, $5 \% \mathrm{CO}_{2}$, and $90 \%$ relative humidity) for $20-24 \mathrm{~h}$. The cells were co-transfected with the plasmid DNAs expressing H-DDX56 and influenza A virus NS1 protein and incubated for $48 \mathrm{~h}$. Furthermore, HEK293 cells were transfected with pCHA-DDX56 plasmid and the cells were infected at $40 \mathrm{~h}$ post-transfection with influenza A/WSN/33/H1N1 virus at one MOI and incubated for $8 \mathrm{~h}$. After the incubation periods, 
transfected or virus-infected cells were washed with PBS and treated with $1 \mathrm{mM}$ DSP (dithiobis succinimidyl propionate) for $1 \mathrm{~h}$. Cross-linking reaction was stopped by the addition of glycine at $100 \mathrm{mM}$ final concentration, and then the cells were lysed in buffer A (50 mM Tris- $\mathrm{HCl}$ pH 8.0, $150 \mathrm{mM} \mathrm{NaCl}, 1$ mM DTT, and $0.1 \%$ NP-40), and the lysates were clarified by centrifugation at $10000 \mathrm{rpm}$ for $5 \mathrm{~min}$. The cell lysates $(300$ $\mu \mathrm{L})$ were incubated with $5 \mu \mathrm{L}$ monoclonal mouse anti-HA antibody (sc-7392) at $4{ }^{\circ} \mathrm{C}$ for $2 \mathrm{~h}$. Protein A Sepharose (GE Healthcare, Sweden) was added to lysates and moderately stirred overnight at $4{ }^{\circ} \mathrm{C}$. The beads were washed three times with buffer A. The proteins bound Sepharose beads were recovered by heating the samples at $95{ }^{\circ} \mathrm{C}$ for 5 minutes in SDS-PAGE loading buffer and then analyzed by western blotting.

\section{Immunofluorescence assay}

The localization of H-DDX56 and influenza A virus NS1 proteins in transfected HeLa cells were analyzed with an immunofluorescence assay. HeLa cells grown on coverslips were transfected with the plasmid DNAs. After 36-40 h transfection, the cells were washed three times with PBS, fixed in 3\% paraformaldehyde for $20 \mathrm{~min}$, and permeabilized with $0.1 \% \mathrm{NP}-40$. After blocking in $1 \%$ skim milk for 30 min, the cells were incubated with the primary antibodies (mouse anti-HA and/or rabbit anti-NS1) diluted in 1\% skim milk for $60 \mathrm{~min}$ at room temperature and then washed twice with $0.1 \%$ NP-40 and once with PBS. After a second blocking with $1 \%$ skim milk for $20 \mathrm{~min}$, the cells were incubated with the Alexa-488-conjugated goat anti-mouse IgG and/or Alexa568-conjugated goat anti-rabbit IgG (at 1:300 dilutions in 1\% skim milk) for $60 \mathrm{~min}$. The nuclei of the cells were stained with DAPI (4',6'-diamidino-2-phenylindole). The coverslip was washed in $0.1 \% \mathrm{NP}-40$ and mounted in a media $(0.1 \%$ p-phenylendiamine and $80 \%$ glycerol), and the cells were analyzed using a fluorescence microscope (Olympus BH40, Japan).

\section{siRNA transfection and quantitative real-time PCR analysis}

To understand the potential role of DDX56 on influenza A virus replication, we used RNA interference techniques. The small interfering RNA of DDX56 (siDDX56) (Cat\#1299001/ HSS182589) was purchased from Life Technologies. The HeLa cells $\left(3 \times 10^{5}\right)$ were seeded in six well-plates and incubated under standard culture conditions for 20-24 h. The cells were transfected with 30 pmol siDDX56 or nonsilencing control siRNA (Invitrogen, \# 12935-200) with lipofectamine RNAiMAX (Thermo Fisher Scientific, USA) reagent according to the manufacturer's protocol and incubated for $48 \mathrm{~h}$. After the incubation, total RNA was extracted from siRNA transfected cells for quantitation of the DDX56 transcript with RT-qPCR. Remaining knock-down cells were sub-cultured for influenza A virus infection. Quantitation of the DDX56 transcript in the cells transfected with siRNA was analyzed with RT-qPCR. Total RNA and cDNAs were prepared as described above. Real-time PCR was conducted using the FastStart Universal SYBR Green Master mix (Roche, Germany). Reactions were carried out in triplicate in a total volume of $10 \mu \mathrm{L}$ containing
$2 \mu \mathrm{L}$ of cDNA and $2.5 \mathrm{pM}$ gene-specific primers. Bio-Rad, the CFX96 model real time PCR instrument, was used for quantitative analysis. The cycle conditions included an initial denaturation step at $94{ }^{\circ} \mathrm{C}$ for $5 \mathrm{~min}$, followed by 45 cycles of amplification for $10 \mathrm{~s}$ at $94{ }^{\circ} \mathrm{C}, 30 \mathrm{~s}$ at $58{ }^{\circ} \mathrm{C}$, and $15 \mathrm{~s}$ at 72 ${ }^{\circ} \mathrm{C}$. Fluorescence was quantified during the $58{ }^{\circ} \mathrm{C}$ annealing step, and the product formation was confirmed by melting curve analysis $\left(65^{\circ} \mathrm{C}\right.$ to $\left.95^{\circ} \mathrm{C}\right)$. As an internal control, the level of the housekeeping actin-beta (ACTB) gene transcript was determined. The amounts of the DDX56 were normalized by the amount of actin-beta transcripts. DDX56/831-For and DDX56/959-Rev primer pair and ACTB-For/ACTB-Rev primer pair were used for amplification of the DDX56 and ACTB cDNAs, respectively. The primer sequences used for the RT-qPCR are listed in Table 1.

\section{Influenza A virus growth in DDX56 knock-down cells}

The quantity of M1 protein, one of the most abundant virus structural proteins in the infected cell, was analyzed by western blotting to observe influenza A virus growth in knockdown cells at early stages of replication. Forty-eight hours post-transfection with siRNAs, the cells were sub-cultured into 24 -well plates $\left(2 \times 10^{5} /\right.$ well $)$ and incubated for $24 \mathrm{~h}$. Then the monolayer cultures were infected with human influenza A (WSN) viruses at $1 \mathrm{MOI}$. After the virus adsorption at 37 ${ }^{\circ} \mathrm{C}$ for $30 \mathrm{~min}$, the inoculum was removed, and the cells were maintained in the maintenance medium for $8 \mathrm{~h}$. The cells were lysed in the SDS-PAGE sample buffer and viral structural M1 proteins in the lysates were analyzed with SDS-PAGE/ Western blotting. In late stage of viral growth, the quantity of virus released into the medium in the infected culture was determined by plaque assay. For this, the knock-down cells were seeded in 12 -well plates $\left(4 \times 10^{5} /\right.$ well $)$, cultured for 24 $\mathrm{h}$ and inoculated with influenza A viruses at 0.1 MOI. The inoculum was removed, and the cultures were incubated in the maintenance medium for $24 \mathrm{~h}$ at $37^{\circ} \mathrm{C}$. At 8, 12 and 24 $\mathrm{h}$ post infection, the medium containing released viruses was collected, and the quantity of the viruses in the medium were determined by plaque assays using MDCK cells (Turan et al., 1996).

\section{Over-expression H-DDX56 and viral infection}

To understand the effect of DDX56 on influenza A virus replication, H-DDX56 protein was over-expressed by transient transfection of plasmid encoding this protein, and the effects of H-DDX56 increase on the virus replication was investigated. The HEK293 cells $\left(2.5 \times 10^{5}\right)$ were seeded in 12-well plate and incubated under standard culture conditions for 20-24 h and the pCHA-DDX56 plasmid DNA was transfected with $2 \mu \mathrm{g}$. After $40 \mathrm{~h}$ transfection, the cells were infected with influenza A (WSN) viruses at $1 \mathrm{MOI}$ and incubated for $8 \mathrm{~h}$. Then the cells were lysed in the SDS-PAGE sample buffer. H-DDX56 and viral proteins in lysates were analyzed with SDS-PAGE/ Western blotting.

\section{In silico prediction of NS1 and DDX56 interaction}

The in silico method was used to predict possible interactions between influenza A virus NS1 and human DDX56 protein. The three-dimensional structure of NS1 
and DDX56 was predicted by using I-TASSER algorithm (https://zhanglab.ccmb.med.umich.edu/I-TASSER/) (Zhang, 2008). Interaction models showing the highest binding affinity between two proteins according to their free binding energies were determined by using the top 3D models of NS1 and DDX56 with pyDOCK protein-protein docking method (http:// life.bsc.es/pid/pydock) (Jimenez-Garcia et al., 2013). Finally, the computational analysis of protein interaction domains was performed via Vega ZZ, a molecular modeling program (Pedretti et al., 2002).

\section{Results}

\section{Cellular RNA helicase DDX56 interacts with influenza A virus NS1 protein in yeast cells}

To understand the roles of NS1 in the pathogenesis of influenza A virus, we screened host proteins that interact with NS1 protein using a GAL4-based yeast two-hybrid assay. Following the two-hybrid system protocol, the $\mathrm{pGBD}-\mathrm{NS} 1_{\mathrm{w}}$ plasmid or the pGBD-NS1 $1_{\mathrm{D}}$ plasmid as bait and a human cDNA library cloned into pACT2 were used. The library screening yielded approximately 150 colonies on the SD agar medium (without Ade, His, Leu, and Trp) after three days of growth at $30^{\circ} \mathrm{C}$. The number was reduced to 50 colonies by eliminating false-positive interactors after the $\beta$-galactosidase assay. Plasmids DNA carrying cDNA were isolated from the colonies with high $\beta$-galactosidase activity, and the cDNAs were sequenced. Sequencing results showed that some of the cDNA were not in the codon frame with GAL4 AD. The candidate genes within the codon frame with GAL4 AD were defined with BLAST analysis. The cDNAs were belong to genes coding DEAD (Asp-Glu-Ala-Asp) box helicase 56 (DDX56), neuroguidin, EIF4E binding protein
(NGDN), proteosome subunit beta type 4 (PSMB4), ribosomal protein L29 (RPL29), and small nuclear ribonucleoprotein D1 polypeptide (SNRPD1) (Figures S1-S5). We focused on DDX56, which has been shown to be effective in intracellular replication of various viruses among these proteins (Xu et al., 2011; Xu and Hobman, 2012; Fu et al., 2019a; Fu et al., 2019b) by directly interacting with the viral proteins or indirectly with host proteins.

The analysis indicated that the DDX56 cDNA part of the GAL4-AD-DDX56 fusion gene consists of the sequence coding 141 amino acids residue of the carboxy-terminal part of the DDX56 protein (Figure S1). Therefore, the full size of DDX56 gene consisting of the sequence coding 548 amino acids residue was amplified with reverse transcription-PCR and cloned into the pACT2 yeast two-hybrid plasmid to construct pACT2-DDX56. To check the result of two-hybrid screening and eliminate false-positive interactions, pACT2-DDX56 plasmid encoding AD-DDX56 was re-transformed into PJ69$4 \mathrm{~A}$ yeast strain expressing $\mathrm{NS}_{\mathrm{W}}$ or $\mathrm{NS}_{\mathrm{D}}$ bait protein, and the growth profiles of the double transformants on the SD agar medium (without Ade, His, Leu, and Trp) were determined (Figure 1). The growth of colonies in the selective SD media indicated a positive interaction between DDX56 and human or avian influenza A virus NS1 protein. As expected, the growth of colonies carrying control plasmid (pGBD-C1 or pACT2) was not observed in the selective media. In addition to growth profiles, to confirm the results, we performed $\beta$-galactosidase assay in the same colonies. The results indicated that the $\beta$-galactosidase enzyme activity significantly increased in yeast cells expressing both NS1 bait protein and DDX56 protein (Figure 1). Very low levels of $\beta$-galactosidase activities were detected in the cells transformed with plasmid expressing either NS1 bait protein or DDX56 protein used as control.

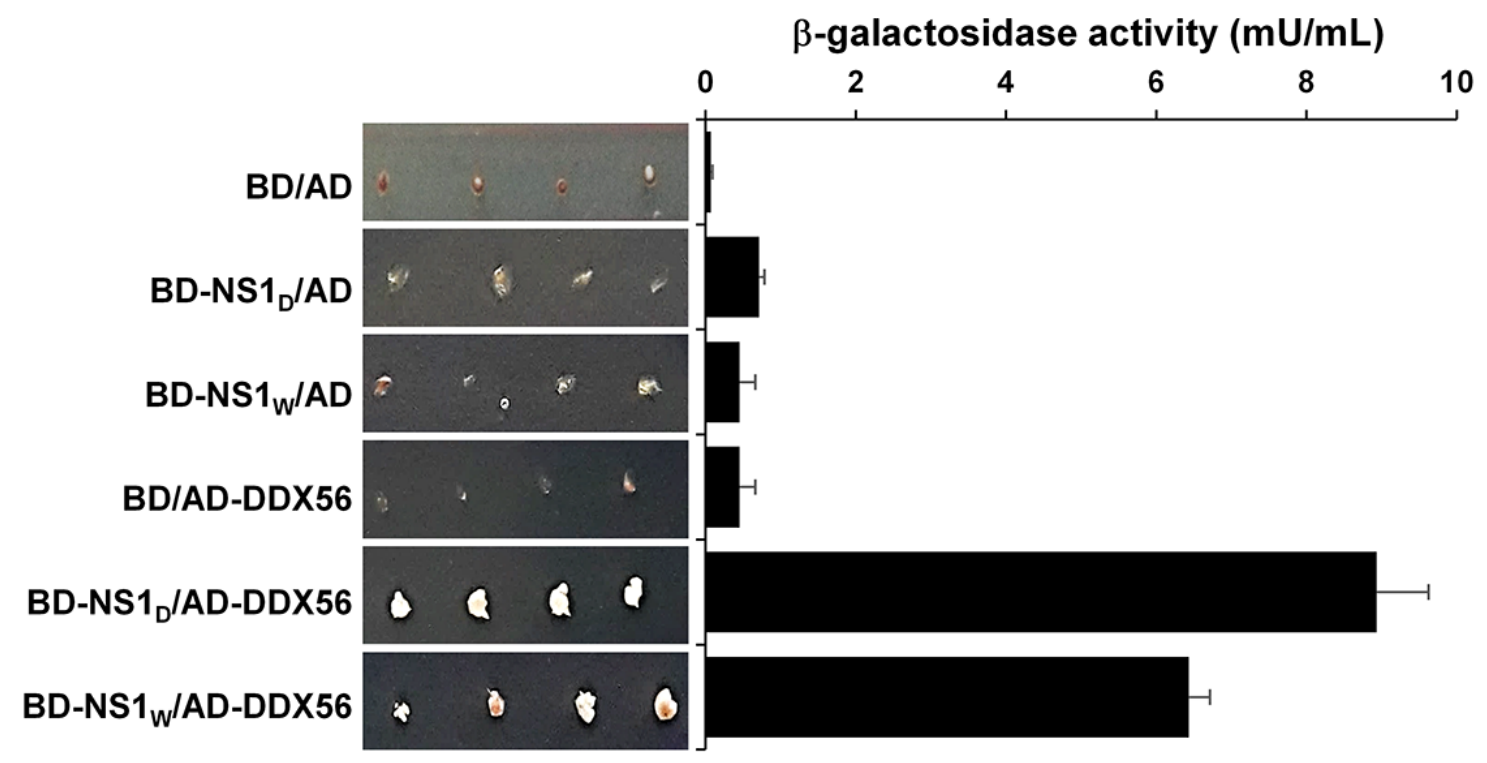

Figure 1 - The growth profiles and $\beta$-galactosidase activity of double transformed $S$. cerevisiae strain PJ69-4A with yeast two-hybrid plasmids. The yeast cells were transformed with a plasmid coding GAL4-binding domain (BD) (pGBD-C1) or viral NS1 bait protein fused with GAL4-BD (pGBD-NS1 or pGBD-NS1 $1_{\mathrm{D}}$ ) and then with a plasmid coding GAL4-activation domain (AD) (pACT2) or human DDX56 protein fused with GAL4-AD (pACT2DDX56). To define the growth profiles, four colonies were cultured on SD agar plates (without Ade, His, Leu, and Trp). The $\beta$-galactosidase enzyme activities of the colonies were defined as described in Methods. 
The DDX56 interacts with influenza A virus NS1 protein in mammalian cells

In order to evaluate the relationship between the influenza A virus NS1 protein and cellular RNA helicase DDX56 having interaction in yeast cells, a co-immunoprecipitation assay and an immunofluorescence assay were carried out in mammalian cells. Therefore, the HEK293 cells were co-transfected with plasmids expressing $\mathrm{H}-\mathrm{DDX} 56$ protein and influenza $\mathrm{A}$ virus NS1 protein $\left(\mathrm{NS}_{\mathrm{w}}\right)$, and the lysates of these cells were subjected to immunoprecipitation with anti-HA antibody against H-DDX56. The immunoprecipitated proteins were analyzed by SDS-PAGE /Western blotting using both mouse anti-HA and rabbit anti-NS1 antibodies. The results showed that the influenza A virus NS1 protein was co-precipitated with H-DDX56 protein. The NS1 protein was not detected in the immunoprecipitates without anti-HA antibody (Figure 2A). The co-immunoprecipitation assay was also carried out with the cells transfected with plasmid DNA coding H-DDX56 protein and then infected with influenza A(WSN). The results showed that viral NS1 protein was also co-precipitated with human DDX56 protein in virus-infected cells (Figure 2B). In order to support the relationship between viral NS1 and human DDX56 protein in the mammalian cells, the cellular localization patterns of these proteins were investigated by immunofluorescence techniques. The results showed that some human DDX56 proteins localize in the nucleus and dominantly in the nucleolus of the cells when expressed alone, while viral NS1 localizes in the cytoplasm (Figure 3A). The human DDX56 proteins localization was not changed in the cells expressing NS1 1 proteins (Figure 3B). In contrast, viral NS1 proteins showed a tendency to co-localize with human H-DDX56 and localized predominantly in the nucleus of the cells expressing both H-DDX56 and viral NS1 proteins, which supports the IP and yeast two-hybrid assays results.

\section{Effects of DDX56 on influenza virus replication}

The effect of human DDX56 protein on influenza A virus replication was evaluated in knock down cells for this gene and in the cells over-expressing H-DDX56 protein with a

A

IB w/anti-HA

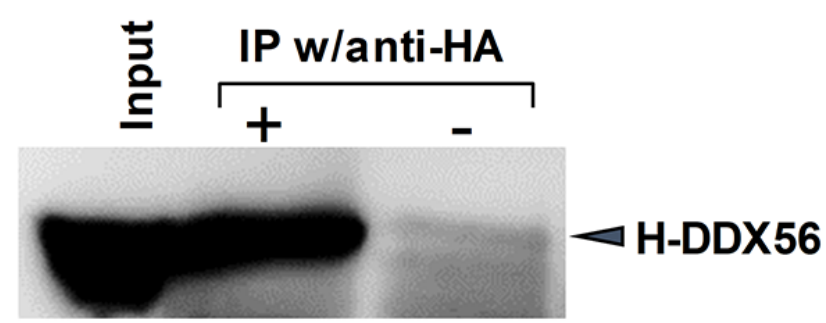

IB w/anti-NS1

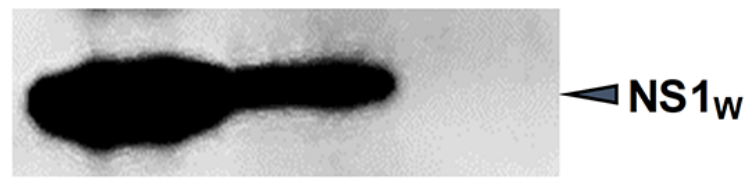

B

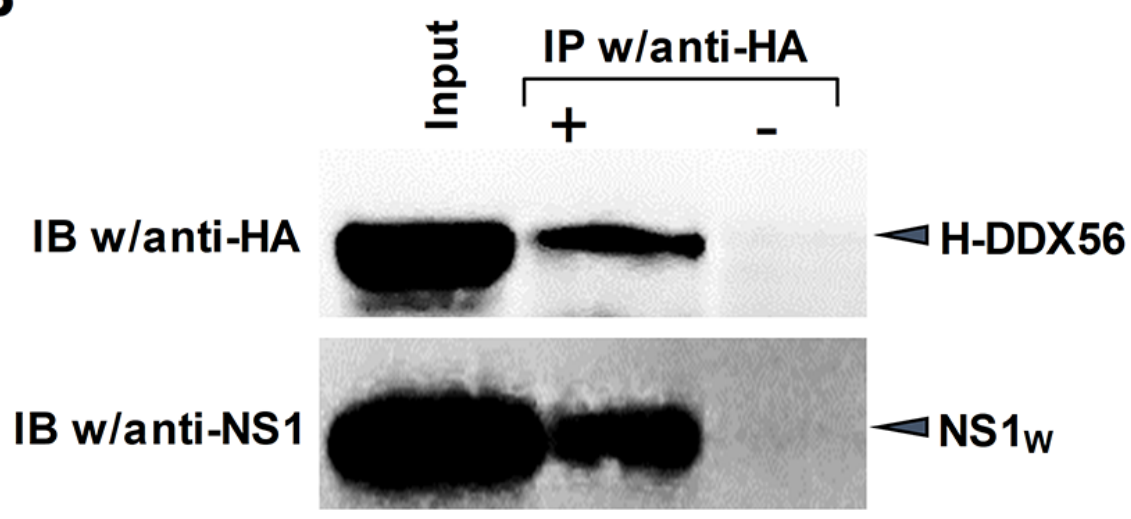

Figure 2 - The immunoprecipitation analysis of human DDX56 and influenza A virus NS1 protein interaction in mammalian cells. The cells were co-transfected with pCHA-DDX56 plasmid and pCAGGS-NS1 $1_{w}$ (A) or transfected with pCHA-DDX56 plasmid and infected with influenza A (WSN) viruses (B). The cell lysates were prepared, and H-DDX56 proteins were precipitated with mouse monoclonal anti-HA antibody as described in the methods section. For blotting rabbit polyclonal anti-NS1 and mouse monoclonal anti-HA antibody were used. 
A
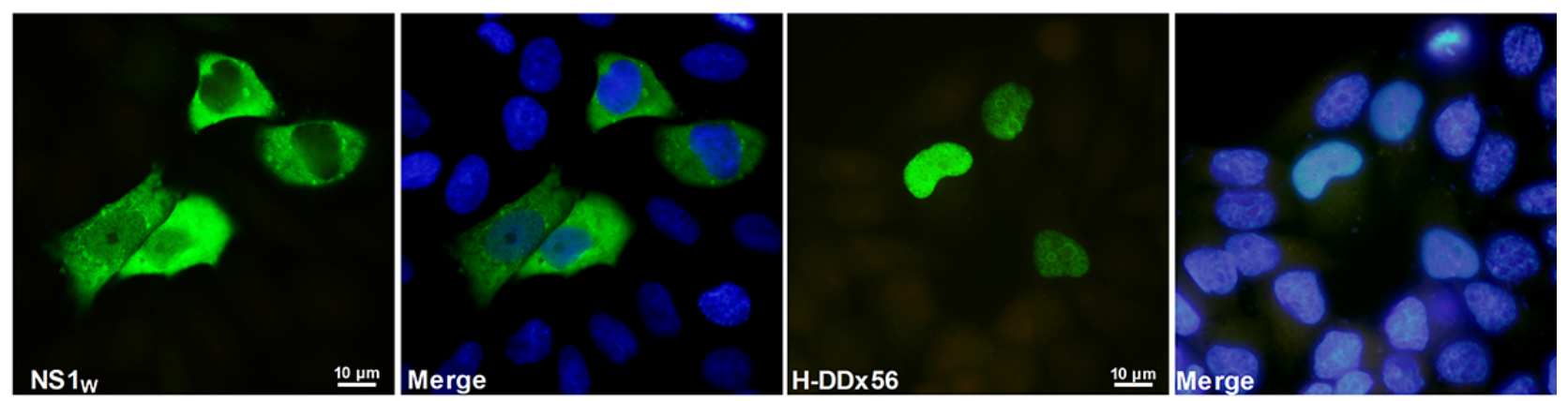

B
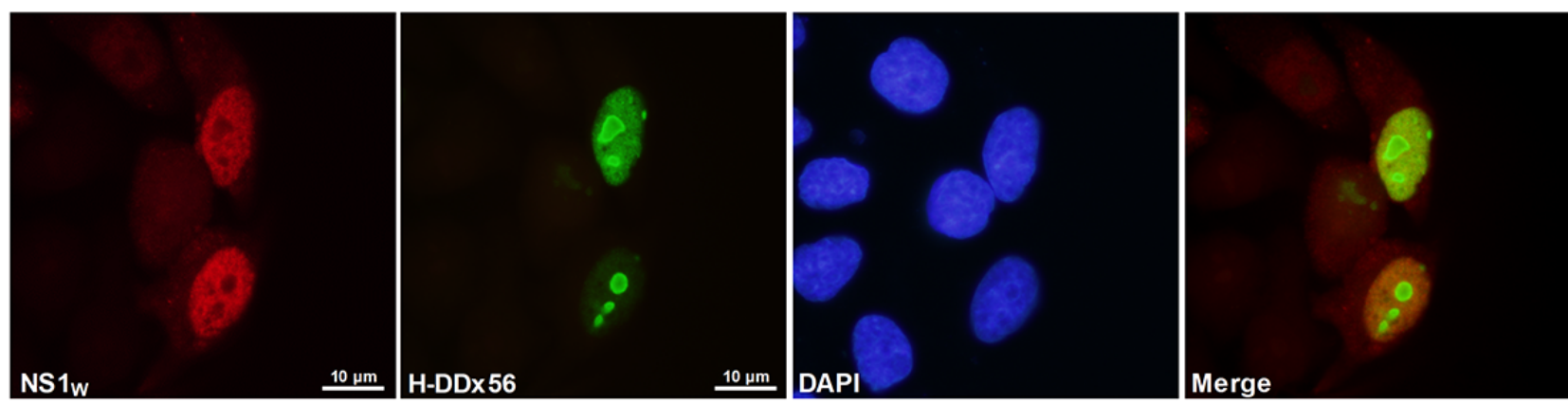

Figure 3 - Localization patterns of influenza A virus NS1 and human DDX56 proteins in HeLa cells transfected with a single plasmid (A) or double plasmid DNAs (B). The cells were fixed with 3\% paraformaldehyde and immunostained with specific antibodies after 36 hours post-transfection. As primary antibodies, mouse monoclonal anti-HA (for H-DDX56) and/or rabbit polyclonal anti-NS1 (for NS1 $1_{w}$ ) were used; as the secondary antibodies, monoclonal anti-mouse IgG conjugated Alexa-488 (for H-DDX56) and the monoclonal anti-rabbit IgG conjugated Alexa-488 or Alexa-568 (for NS1 proteins) were used. The nuclei of the cells were stained with DAPI. The samples were examined with X100 oil immersion objective. Images were captured using a fluorescence microscope (Olympus BH40, Japan).

transient transfection. The DDX56 transcript level was reduced in HeLa cells with transfection of gene-specific siRNA. The quantity of DDX56 transcript normalized with the actin beta gene was detected with RT-qPCR. The data showed that the DDX56 mRNA level was decreased by approximately 90\% (Figure 4A). The knock down cells were infected with human influenza A (WSN) viruses and at an early phase of infection ( $8 \mathrm{~h} \mathrm{p.i.),} \mathrm{and} \mathrm{the} \mathrm{M1} \mathrm{protein} \mathrm{that} \mathrm{is} \mathrm{one} \mathrm{of} \mathrm{the} \mathrm{most}$ abundant viral structural proteins encoded from segment 7 were analyzed by immunoblotting. The influenza A virus M1 protein was found markedly reduced in DDX56-knockdown cells compared with that in the control cells (Figure 4B). The quantity of the viruses released in the medium in infected cultures at the later stages of infection were determined with plaque assay. At 12 and $24 \mathrm{~h}$ post infection, the lower titer of viruses was detected in the cells transfected with DDX56 siRNAs than that of the control cells. (Figure 4C).

In order to see the effect of DDX56 over-expression on the influenza virus replication, HEK293 cells were transfected with the plasmid encoding this protein and then infected with human influenza A (WSN) viruses. At $8 \mathrm{~h}$ p.i. the cells were lysed in SDS-sample buffer, and the viral M1 protein was analyzed with Western blotting. As shown in Figure 4D, the influenza A virus replication was not significantly changed in the HEK293 cells, overexpressing DDX56 compared to the control cells. The data obtained from the cells overexpressing the DDX56 and knockdown cells suggests that the DDX56 protein has a positive regulatory effect on influenza A virus replication.

\section{In silico prediction of NS1 and DDX56 interaction}

Since the native structures are unknown, the tertiary structures of full-size human DDX56 and influenza NS1 proteins were predicted by using the I-TASSER algorithm from on line data server. The C-scores and 3D structures of the five top models are given in Table 2 and Figure S6 and $\mathrm{S} 7$, respectively.

The interactions between model NS1-1 and model DDX56-1, the top models determined with the I-TASSER algorithm, were predicted with the pyDOCK protein-protein docking method (Jimenez-Garcia et al., 2013). Three predicted interaction models with the highest binding affinity (with lower Gibbs' free energy of binding/ $\Delta \mathrm{G}$ ) were analyzed with the Vega ZZ modeling program. In these interaction models, the NS1 protein was found to bind to the carboxyl terminal region of the DDX56 protein (Figure 5). These results support the findings of the yeast two hybrid assay that showed the interaction of the carboxyl terminal domain (last 141 amino acid residues) of human DDX56 with viral NS1 protein. 
A

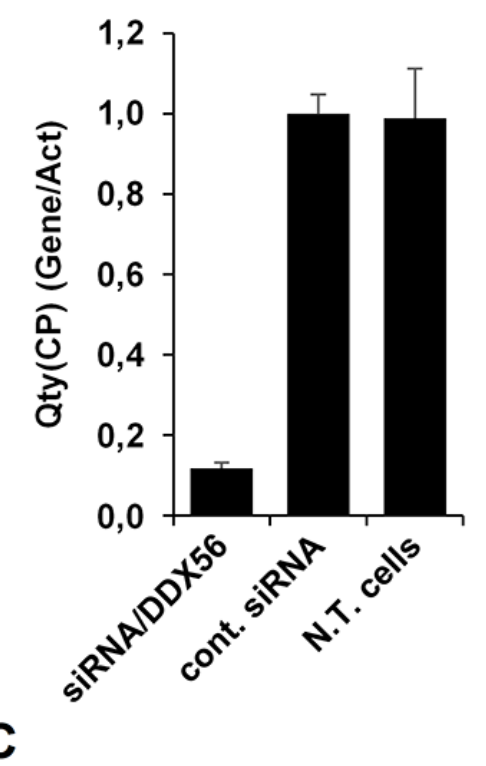

B

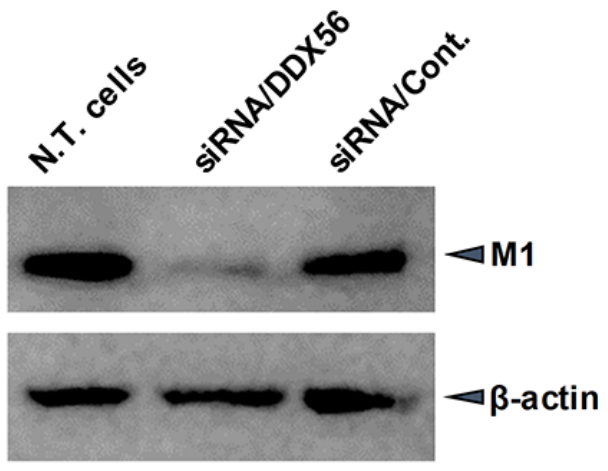

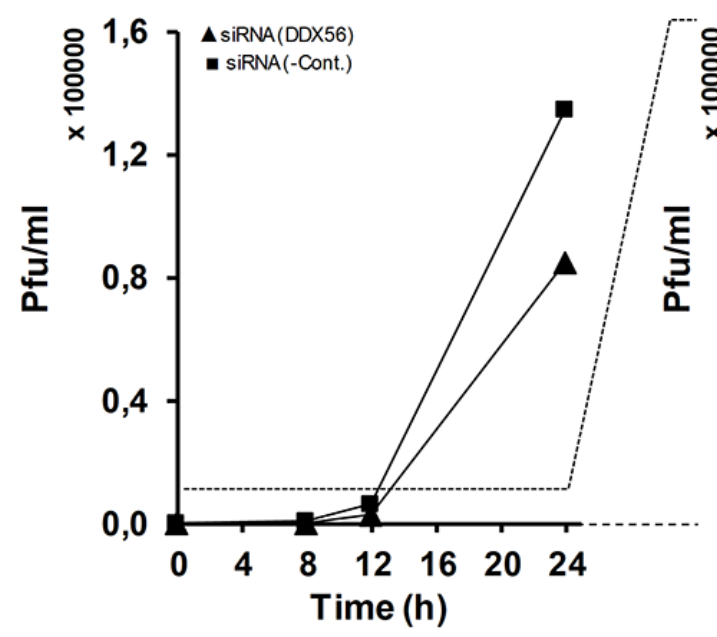

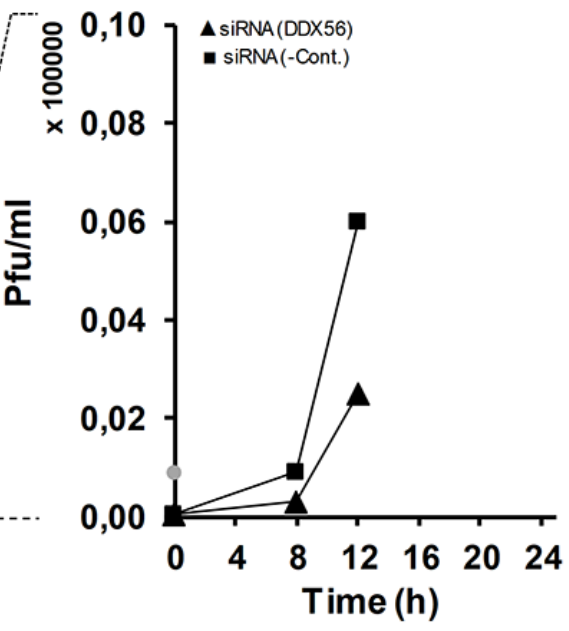

D
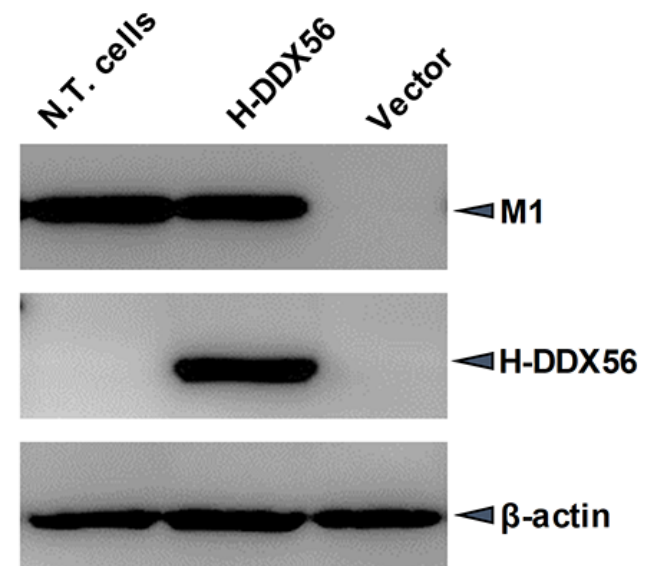

Figure 4 - The quantitation of human DDX56 transcript and viral M1 protein in the cells transfected with siRNA or H-DDX56 encoding plasmid DNA (pCHA-DDX56) and infected with influenza A viruses. A. Quantity of human DDX56 transcript in the non-transfected HeLa cells (N.T. cells), the HeLa cells transfected with DDX56 specific siRNA (siRNA/DDX56), and negative control siRNA (cont.siRNA). B. Viral M1 protein quantity in HeLa cells transfected with siRNAs and then infected with viruses. C. The quantity (plaque forming unit; pfu/ml) of influenza A virus in medium of knock down/ infected cell culture. D. The M1 protein quantity in non-transfected HEK293 cells (N.T. cells) and in HEK293 cells transfected with pCHA-DDX56 or pCHA (Vector) plasmid and then infected with human influenza A (WSN) viruses. After $8 \mathrm{~h}$ p.i. the cells harvested and the proteins in the cell lysates were separated on $10 \%$ PAGE. As primary antibodies, mouse monoclonal anti-HA, rabbit polyclonal anti-M1 serum, and mouse monoclonal anti-actin beta antibodies were used. 
Table 2 - C-scores for predicted 5 top NS1 and DDX56 models

\begin{tabular}{|c|c|c|c|c|c|}
\hline Models & C-score & Exp.TM-Score & Exp.RMSD & No.of decoys & Cluster density \\
\hline NS1-1 & -0.59 & $0.64 \pm 0.13$ & $6.9 \pm 4.1$ & 6410 & 0.1134 \\
\hline NS1-2 & -1.17 & & & 3329 & 0.0633 \\
\hline NS1-3 & -1.36 & & & 2890 & 0.0523 \\
\hline NS1-4 & -2.99 & & & 4465 & 0.0102 \\
\hline NS1-5 & -0.80 & & & 4197 & 0.0918 \\
\hline DDX56-1 & -1.05 & $0.58 \pm 0.14$ & $10.0 \pm 4.6$ & 1165 & 0.0841 \\
\hline DDX56-2 & -1.44 & & & 741 & 0.0573 \\
\hline DDX56-3 & -2.27 & & & 318 & 0.0249 \\
\hline DDX56-4 & -3.22 & & & 134 & 0.0096 \\
\hline DDX56-5 & -2.31 & & & 362 & 0.0239 \\
\hline
\end{tabular}

model 1
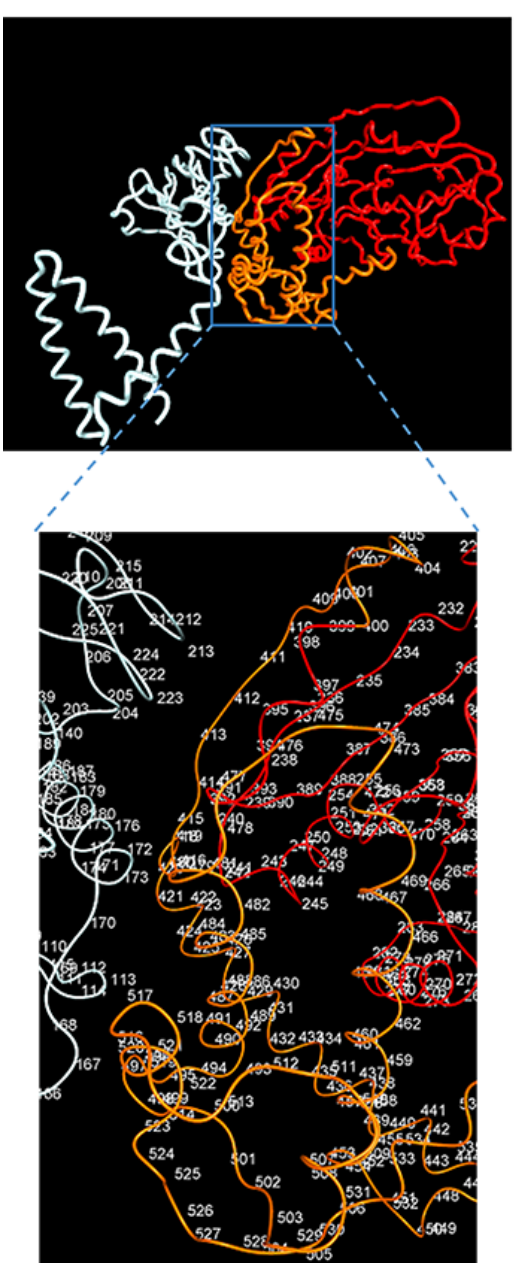

model 2
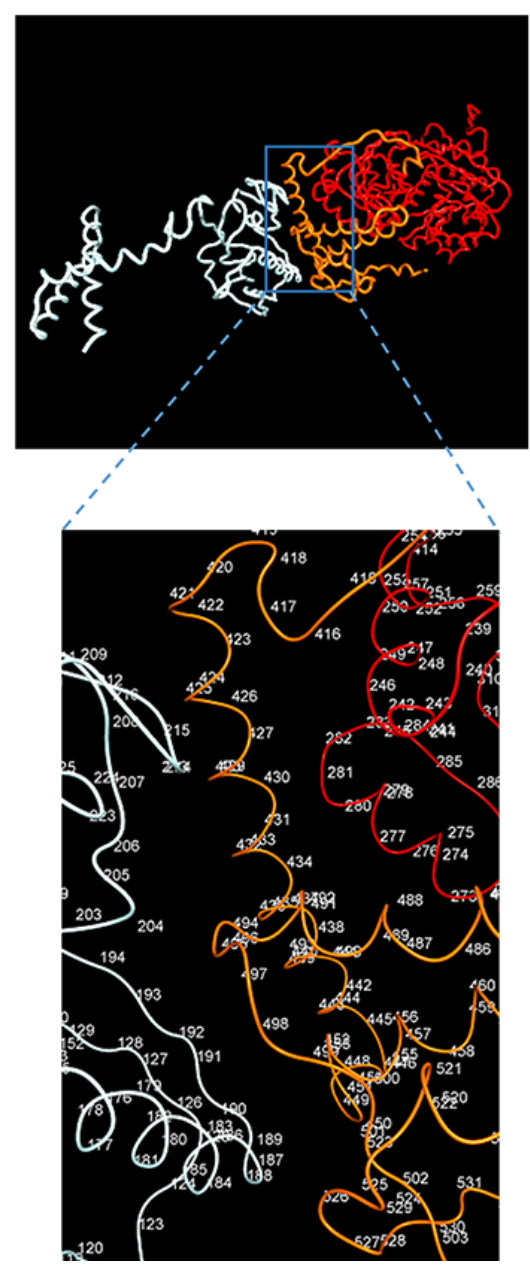

model 3
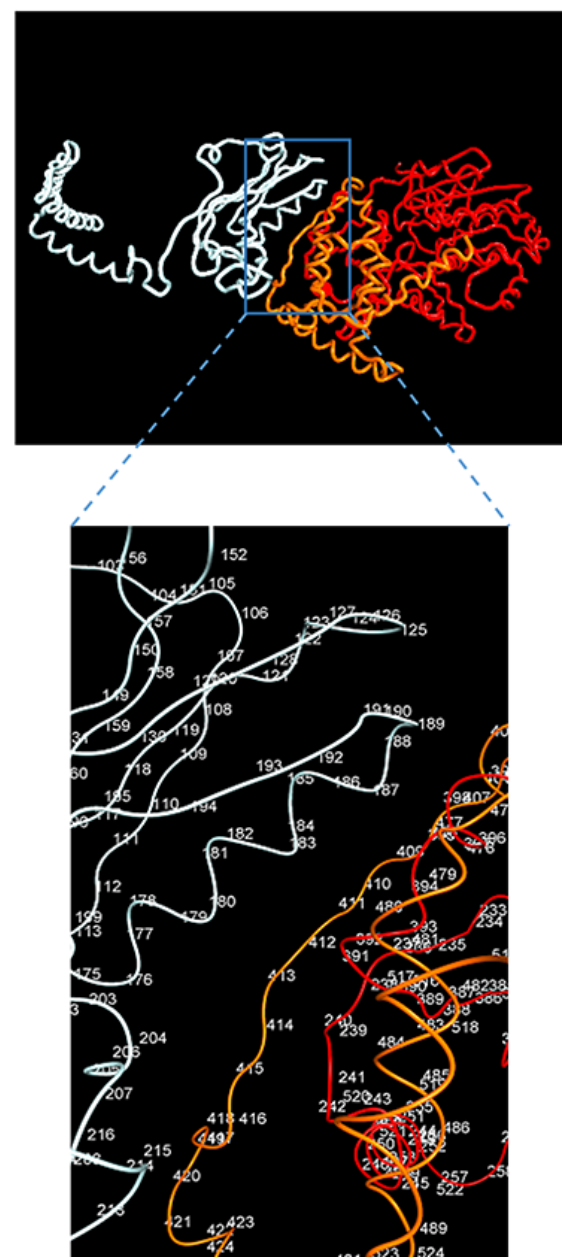

Figure 5 - In silico models of the influenza A virus NS1 and human DDX56 protein interaction. The predicted ternary structures of NS1(white) and human DDX56 (red and orange) proteins are shown. In the lower panel, the predicted interface of NS1 and DDX56 proteins are given as enlarged and numbered amino acid residues. The 3D-structures and the interaction models of the proteins were obtained using I-TASSER algorithm and Pymol software, respectively. The Gibbs free energy of binding $(\Delta \mathrm{G})$ for interactions: -56.267 (model 1), -50.429 (model 2), -47.185 (model 3).

\section{Discussion}

One of the important properties of influenza A viruses that distinguishes it from the other RNA viruses is the necessity to transport viral genome components into the host cell nuclei for replication and transcription. The main reason for this is that the viral RNA polymerase enzyme is dependent on the cellular RNA polymerase II enzyme (Engelhardt et al., 2005). Influenza A virus RNA dependent RNA polymerase (RdRP) removes the 5' cap structure of pre-mRNAs synthesized by RNA pol II (cap snatching activity) and uses it in the transcription of viral mRNAs (Dias et al., 2009). Therefore, the nucleo-cytoplasmic traffic of the influenza virus components in the host cells is much more intense compared to the other RNA viruses. Several cellular protein factors involved in influenza $\mathrm{A}$ virus replication and the nucleo-cytoplasmic traffic of viral components were identified (Senbas Akyazi et al., 2020). In order to understand 
the influenza A virus replication strategies, it is very important to reveal the relationship between cellular and viral factors at the molecular level. Some of the host cellular protein factors interfering with influenza A virus replication regulate the viral replication positively while others have negative effects (Turan et al., 2004; Gao et al., 2015). In this study, the new host proteins that may be related to the viral NS1 protein which functions to eliminate the host interferon response to influenza A viruses (Gao et al., 2012) was screened with the yeast two-hybrid method, and several candidate protein factors as NS1 interactor were determined (Figures S1-S5). Among these proteins, the relationship of human DDX56 protein (as also known NOH61) (Zirwes et al., 2000) with the viral NS1 protein in both yeast and mammalian cells and the effects of this protein on influenza A virus replication were investigated. The interaction between these two proteins was also evaluated with in silico methods. The yeast two-hybrid analysis was repeated with pACT2-DDX56 constructed by cloning the complete cDNA of DDX56. The growth profiles of double transformants were defined on the selective medium for the primary screening and then tested by the $\beta$-galactosidase assay. The results showed that both human and avian influenza A virus NS1 proteins and DDX56 protein have an interaction in yeast cells (Figure 1).

The DDX56 protein is a member of the DEAD box protein family with ATP-dependent RNA helicase activity. All DEAD-box proteins contain a highly conserved helicase core that consists of two RecA-like domains with at least 12 conserved amino acid motifs. The helicase core has ATP binding, RNA binding, and RNA duplex unwinding activities (Linder et al., 1989; Cordin et al., 2006). The DEAD-box helicases are identified as 37 members in human and 26 in $S$. cerevisiae. One of them, the human DDX56 (yeast homologue Dbp9), mainly functions during ribosome biogenesis and the assembly of $60 \mathrm{~S}$ ribosomal subunits in the nucleolus as well as in all biological processes where RNA plays a central role such as transcription, mRNA splicing, mRNA transport, translation, and mitochondrial gene expression. Recent studies described how RNA helicases participate in viral infections. Some RNA viruses carry their own helicases and use them for a replication process such as HCV NS3. Modulating the nuclear-cytoplasmic transport is essential for $\mathrm{HCV}$ replication (Gu and Rice, 2010). On the other hand, most RNA viruses do not encode their own helicase and use the cellular RNA helicases to support their life cycle, such as HIV-I, WNV, and IAV. Some cellular helicases enhance viral replication like a co-factor. During HIV-I infection, DDX1 and DDX5/p68 enhance the Rev-mediated nuclear export of HIV-1 transcripts (Yasuda-Inoue et al., 2013). In contrast, some cellular helicases play central roles in the antiviral defense mechanism. During HBV infection, DDX3 restricts viral replication by binding to HBV polymerase and inhibiting reverse transcription (Wang $\mathrm{H}$ et al., 2009). Therefore, we speculated that the cellular RNA helicase DDX56 could affect influenza A virus replication by interacting with viral NS1 protein in infection.

The yeast two-hybrid method is an effective technique in determining the relationship between proteins and protein functions, but it is an artificial system and may not fully reflect the native conditions. Therefore, the relationship between influenza A virus NS1 and human DDX56 proteins and the effects of DDX56 protein on influenza virus replication were evaluated from mammary cells. The open reading frames of human DDX56 and influenza A virus NS1 genes were cloned in the mammalian expression plasmids (pCAGGS or pCHA), and the plasmid vectors were used for transient transfection of HEK293 and HeLa cells. The immunoprecipitation and immunofluorescence techniques were applied to reveal the relationship between human DDX56 and influenza A virus NS1 protein synthesized in virus infected and/or transiently transfected cells. For the co-immunoprecipitation assays, HEK293 cells were co-transfected with plasmids expressing H-DDX56 protein and influenza A virus NS1 protein. We also tested whether viral NS1 protein could interact with DDX56 protein in virus infected cells. For this aim, HEK293 cells were transfected with pCHA-DDX56 plasmid vector and then infected with influenza A (WSN) viruses. The cell lysates were subjected to co-IP with anti-HA antibody. The results showed that the viral NS1 protein co-precipitated with H-DDX56, suggesting that human DDX56 and influenza A virus NS1 protein interact in mammalian cells (Figure 2). The intracellular localization patterns of the proteins also give important clues about the interaction between proteins. The co-localization tendency of viral NS1 proteins with H-DDX56 protein in the nuclei of transiently transfected HeLa cells supports the IP results (Figure 3).

In several reports, although not related with the influenza A viruses, it was shown that RNA helicase DDX56 interferes with some virus replication. For instance, DDX56 has an up-regulator effect on FMDV replication and co-expression DDX56, and FMDV 3A protein inhibits the type I interferon by reducing the phosphorylation of IRF3 (Fu et al., 2019a). On the other hand, DDX56 is not important for the replication of WNV. Instead, DDX56 interacts with WNV capsid protein and plays an essential role in the assembly of infectious WNV virions (Xu et al., 2011; Xu and Hobman, 2012). Furthermore, during WNV infection, the nucleolar helicase DDX56 changes localization to virus assembly sites on the endoplasmic reticulum (Reid and Hobman, 2017). In addition to DDX3 and DDX5, DDX56 enhances Rev-dependent RNA export function of the unspliced/partially spliced HIV-1 mRNAs during HIV-I infection (Yasuda-Inoue et al., 2013).

In this study, the interaction of influenza A virus NS1 and human DDX56 proteins, both in yeast and mammalian cells, was shown. In order to reveal the effects of DDX56 protein on influenza $A$ viruses, the viral replication in the cells knocked down with specific siRNA for DDX56 transcript or over-expressed DDX56 protein was monitored with immunoblotting. The influenza A virus replication was downregulated in the cells transfected with DDX56 specific siRNA (Figure 4B and 4C), showing that the DDX56 proteins have stimulatory effects on influenza A virus replication. In contrast, no significant changes were observed in the quantity of viral M1 proteins in the virus infected cells over-expressing DDX56 (Figure 4D). From this result, it was concluded that the endogenous DDX56 protein are sufficient for viral replication. The significant decrease of viral M1 protein in virus infected knock down cells indicates that DDX56 protein has a positive regulatory effect on influenza A virus replication. However, 
the action mechanism of DDX56 on virus replication and the role of NS1 in this event are not clearly understood. It has been reported that DDX56 has a negative regulatory effect on virus-triggered type I IFN induction by disrupting the interaction between IRF3 and IPO5, which inhibits the nuclear translocation of IRF3 (Li et al., 2020). The positive regulator effect of DDX56 on influenza A virus replication may be due to the disruption of IFN induction. Another speculation to explain the effect of DDX56 on viral replication is that DDX56 and NS1 may cooperate for remodeling of the secondary structure of viral RNAs and enhance the viral RNA polymerase activity.

In the yeast cells, the carboxyl terminal region consisting of 141 amino acid residues of DDX56 protein fused with GAL4 $\mathrm{AD}$ interacts with viral NS1 protein (Figure S1). In silico predicted protein interface models also support the binding of DDX56 protein to NS1 protein via carboxyl terminal region (Figure 5). From these results, it was concluded that the carboxyl terminal region of the DDX56 is important for interaction with NS1 protein. However, the action mechanism of human DDX56 on influenza A virus replication requires further elucidation.

In conclusion, it has been shown that the human DDX56 protein, ATP-dependent RNA helicase, interacts with influenza A virus NS1 protein in both yeast and mammalian cells and has a positive regulatory effect on viral replication.

\section{Acknowledgments}

This work was supported by a grant from the Marmara University Research Foundation (grant no: SAG-C-DRP081117-0617) and by the Scientific and Technological Research Council of Turkey (TÜBİTAK, grant no: SBAG-112S518).

\section{Conflict of Interest}

The authors declare that there is no conflict of interest that could be perceived as prejudicial to the impartiality of the reported research.

\section{Author Contributions}

KT conceived the study, AP conducted the experiments, AP and KT analyzed the data, and AP and KT wrote the manuscript. All authors read and approved the final version.

\section{References}

Basu D, Walkiewicz MP, Frieman M, Baric RS, Auble DT and Engel DA (2009) Novel influenza virus NS1 antagonists block replication and restore innate immune function. J Virol 83:1881-1891.

Burgui I, Aragon T, Ortin J and Nieto A (2003) PABP1 and eIF4GI associate with influenza virus NS1 protein in viral mRNA translation initiation complexes. J Gen Virol 84:3263-3274.

Cordin O, Banroques J, Tanner NK and Linder P (2006) The DEADbox protein family of RNA helicases. Gene 367:17-37.

de la Luna S, Fortes P, Beloso A and Ortin J (1995) Influenza virus NS1 protein enhances the rate of translation initiation of viral mRNAs. J Virol 69:2427-2433.

Dias A, Bouvier D, Crepin T, McCarthy AA, Hart DJ, Baudin F, Cusack S and Ruigrok RW (2009) The cap-snatching endonuclease of influenza virus polymerase resides in the PA subunit. Nature 458:914-918.
Engelhardt OG, Smith M and Fodor E (2005) Association of the influenza A virus RNA-dependent RNA polymerase with cellular RNA polymerase II. J Virol 79:5812-5818.

Fu SZ, Yang WP, Ru Y, Zhang KS, Wang Y, Liu XT, Li D and Zheng HX (2019a) DDX56 cooperates with FMDV 3A to enhance FMDV replication by inhibiting the phosphorylation of IRF3. Cell Signal 64:109393.

Fu SZ, Li L, Zhang J, Li D and Zheng HX (2019b) Porcine DDX56 regulates the Foot and Mouth disease virus replication and the virus-triggered RLR pathway. Acta Vet Zoot Sinica 50:18491856.

Gao S, Song L, Li J, Zhang Z, Peng H, Jiang W, Wang Q, Kang T, Chen S and Huang W (2012) Influenza A virus-encoded NS1 virulence factor protein inhibits innate immune response by targeting IKK. Cell Microbiol 14:1849-1866.

Gao S, Wu J, Liu RY, Li J, Song L, Teng Y, Sheng C, Liu D, Yao C, Chen H et al. (2015) Interaction of NS2 with AIMP2 facilitates the switch from ubiquitination to SUMOylation of M1 in influenza A virus-infected cells. J Virol 89:300-311.

Gu M and Rice CM (2010) Three conformational snapshots of the hepatitis $\mathrm{C}$ virus NS3 helicase reveal a ratchet translocation mechanism. Proc Natl Acad Sci U S A 107:521-528.

Hale BG, Randall RE, Ortin J and Jackson D (2008) The multifunctional NS1 protein of influenza A viruses. J Gen Virol 89:2359-2376

Hatada E and Fukuda R (1992) Binding of influenza A virus NS1 protein to dsRNA in vitro. J Gen Virol 73:3325-3329.

James P, Halladay J and Craig EA (1996) Genomic libraries and a host strain designed for highly efficient two-hybrid selection in yeast. Genetics 144:1425-1436.

Jimenez-Garcia B, Pons C and Fernandez-Recio J (2013) pyDockWEB: a web server for rigid-body protein-protein docking using electrostatics and desolvation scoring. Bioinformatics 29:1698-1699.

Kawaoka Y and Neumann G (2012) Influenza viruses: an introduction. Methods Mol Biol 865:1-9.

Kochs G, Garcia-Sastre A and Martinez-Sobrido L (2007) Multiple anti-interferon actions of the influenza A virus NS1 protein. J Virol 81:7011-7021.

Lam WY, Yeung AC and Chan PK (2011) Apoptosis, cytokine and chemokine induction by non-structural 1 (NS1) proteins encoded by different influenza subtypes. Virol J 8:554.

Lamb RA and Choppin PW (1979) Segment 8 of the influenza virus genome is unique in coding for two polypeptides. Proc Natl Acad Sci U S A 76:4908-4912.

Li D, Fu S, Wu Z, Yang W, Ru Y, Shu H, Liu X and Zheng H (2020) Correction: DDX56 inhibits type I interferon by disrupting assembly of IRF3-IPO5 to inhibit IRF3 nucleus import. J Cell Sci 133:jes244681.

Lin D, Lan J and Zhang Z (2007) Structure and function of the NS1 protein of influenza A virus. Acta Biochim Biophys Sin (Shanghai) 39:155-162.

Linder P, Lasko PF, Ashburner M, Leroy P, Nielsen PJ, Nishi K, Schnier J and Slonimski PP (1989) Birth of the D-E-A-D box. Nature 337:121-122.

Nagata K, Saito S, Okuwaki M, Kawase H, Furuya A, Kusano A, Hanai N, Okuda A and Kikuchi A (1998) Cellular localization and expression of template-activating factor I in different cell types. Exp Cell Res 240:274-281.

Nakajima K (1997) Influenza virus genome structure and encoded proteins. Nihon Rinsho 55:2542-2546.

Nemeroff ME, Barabino SM, Li Y, Keller W and Krug RM (1998) Influenza virus NS1 protein interacts with the cellular $30 \mathrm{kDa}$ subunit of CPSF and inhibits 3'end formation of cellular premRNAs. Mol Cell 1:991-1000. 
Neumann G, Hughes MT and Kawaoka Y (2000) Influenza A virus NS2 protein mediates vRNP nuclear export through NESindependent interaction with hCRM1. EMBO J 19:6751-6758.

Newby CM, Sabin L and Pekosz A (2007) The RNA binding domain of influenza A virus NS1 protein affects secretion of tumor necrosis factor alpha, interleukin-6, and interferon in primary murine tracheal epithelial cells. J Virol 81:9469-9480.

Niwa H, Yamamura K and Miyazaki J (1991) Efficient selection for high-expression transfectants with a novel eukaryotic vector. Gene 108:193-199.

Qiu Y, Nemeroff M and Krug RM (1995) The influenza virus NS1 protein binds to a specific region in human U6 snRNA and inhibits U6-U2 and U6-U4 snRNA interactions during splicing. RNA 1:304-316.

Pedretti A, Villa L, Vistoli G (2002) VEGA: a versatile program to convert, handle and visualize molecular structure on Windowsbased PCs. J Mol Graph Model 21:47-49.

Reid CR and Hobman TC (2017) The nucleolar helicase DDX56 redistributes to West Nile virus assembly sites. Virology 500:169-177.

Senbas Akyazi B, Pirincal A, Kawaguchi A, Nagata K and Turan K (2020) Interaction of influenza A virus NS2/NEP protein with the amino-terminal part of Nup214. Turk J Biol 44:82-92.

Stasakova J, Ferko B, Kittel C, Sereinig S, Romanova J, Katinger $\mathrm{H}$ and Egorov A (2005) Influenza A mutant viruses with altered NS1 protein function provoke caspase-1 activation in primary human macrophages, resulting in fast apoptosis and release of high levels of interleukins 1beta and 18. J Gen Virol 86:185-195.

Turan K, Nagata K and Kuru A (1996) Antiviral effect of Sanicula europaea L. leaves extract on influenza virus-infected cells. Biochem Biophys Res Commun 225:22-26.

Turan K, Mibayashi M, Sugiyama K, Saito S, Numajiri A and Nagata K (2004) Nuclear MxA proteins form a complex with influenza virus NP and inhibit the transcription of the engineered influenza virus genome. Nucleic Acids Res 32:643-652

Wang H, Kim S and Ryu WS (2009) DDX3 DEAD-Box RNA helicase inhibits hepatitis B virus reverse transcription by incorporation into nucleocapsids. J Virol 83:5815-5824.

Wang W, Riedel K, Lynch P, Chien CY, Montelione GT and Krug RM (1999) RNA binding by the novel helical domain of the influenza virus NS1 protein requires its dimer structure and a small number of specific basic amino acids. RNA 5:195-205.

Xia S, Monzingo AF and Robertus JD (2009) Structure of NS1A effector domain from the influenza A/Udorn/72 virus. Acta Crystallogr D Biol Crystallogr 65:11-17.

Xu Z and Hobman TC (2012) The helicase activity of DDX56 is required for its role in assembly of infectious West Nile virus particles. Virology 433:226-235.
Xu Z, Anderson R and Hobman TC (2011) The capsid-binding nucleolar helicase DDX56 is important for infectivity of West Nile virus. J Virol 85:5571-5580.

Yasuda-Inoue M, Kuroki M and Ariumi Y (2013) Distinct DDX DEAD-box RNA helicases cooperate to modulate the HIV-1 Rev function. Biochem Biophys Res Commun 434:803-808.

Zhang Y (2008) I-TASSER server for protein 3D structure prediction. BMC Bioinformatics 9:40.

Zirwes RF, Eilbracht J, Kneissel S and Schmidt-Zachmann MS (2000) A novel helicase-type protein in the nucleolus: protein NOH61. Mol Biol Cell 11:1153-1167.

\section{Internet Resources}

I-TASSER, https://zhanglab.ccmb.med.umich.edu/I-TASSER/ (accessed 26 September 2020)

pyDockWEB, http://life.bsc.es/pid/pydock. (accessed 24 October 2020)

\section{Supplementary material}

The following online material is available for this article:

Figure S1 - The sequencing chromatogram (A) and BLAST analysis (B) of DEAD (Asp-Glu-Ala-Asp) box helicase 56 (DDX56).

Figure S2 - The sequencing chromatogram (A) and BLAST analysis (B) of neuroguidin, EIF4E binding protein (NGDN). Figure S3 - The sequencing chromatogram (A) and BLAST analysis (B) of proteasome subunit beta type 4 (PSMB4).

Figure S4 - The sequencing chromatogram (A) and BLAST analysis (B) of ribosomal protein L29 (RPL29).

Figure S5 - The sequencing chromatogram (A) and BLAST analysis (B) of small nuclear ribonucleoprotein D1 polypeptide (SNRPD1).

Figure S6 - The top five I-TASSER 3D models of human DDX56 protein.

Figure S7 - The top five I-TASSER 3D models of influenza A virus NS1 protein.

Associate Editor: Célia Maria de Almeida Soares

License information: This is an open-access article distributed under the terms of the Creative Commons Attribution License (type CC-BY), which permits unrestricted use, distribution and reproduction in any medium, provided the original article is properly cited. 